\title{
Modelling Nonlinear Site Effects in Physics-Based Ground Motion Simulation
}

Christopher A. de la Torre \& Brendon A. Bradley

Department of Civil \& Natural Resources Engineering, University of Canterbury christopher.delatorre@pg.canterbury.ac.nz

1. Background

This study examines the performance of site response analysis via nonlinear total-stress 1D wave-propagation for modelling site effects in physics-based ground motion phenomena at the regional scale, as well as detailed nonlinear site effects at the local scale. The approach is compared to a more commonly used empirical $V_{s 30}$ (30 $\mathrm{m}$ time-averaged shear wave velocity)-based method for computing site amplification as proposed by Graves and Pitarka $(2010$, 2015) and to empirical ground motion prediction via a ground motion model (GMM).

\section{Site Response Analysis Methodologies}

Empirical $\mathbf{V}_{\mathbf{S 3 0}}$-Based Method: Figure 1a shows period-dependent nonlinear site amplification factors from the empirical ground motion mode (GMM) by Campbell and Bozorgnia (2014). This function is then truncated, as recommended by Graves and Pitarka (2010), for two different reasons: 1) long should arce truncated because the 3D long period component of the sim and 2) short periods are trucated because this amplification function is me to be applied to response spectra, but in this context it is applied to Fourier spectra in the frequency domain.

Physics-Based Wave Propagation Analysis: Figure 1b illustrates physics-based site response via wave propagation, in which simulated ground motions are extracted from the 3D model, deconvolved, and used as input to a viscoelastic, they can be deconvolved in the frequency domain using a transfer function for damped soil over an elastic halfspace.
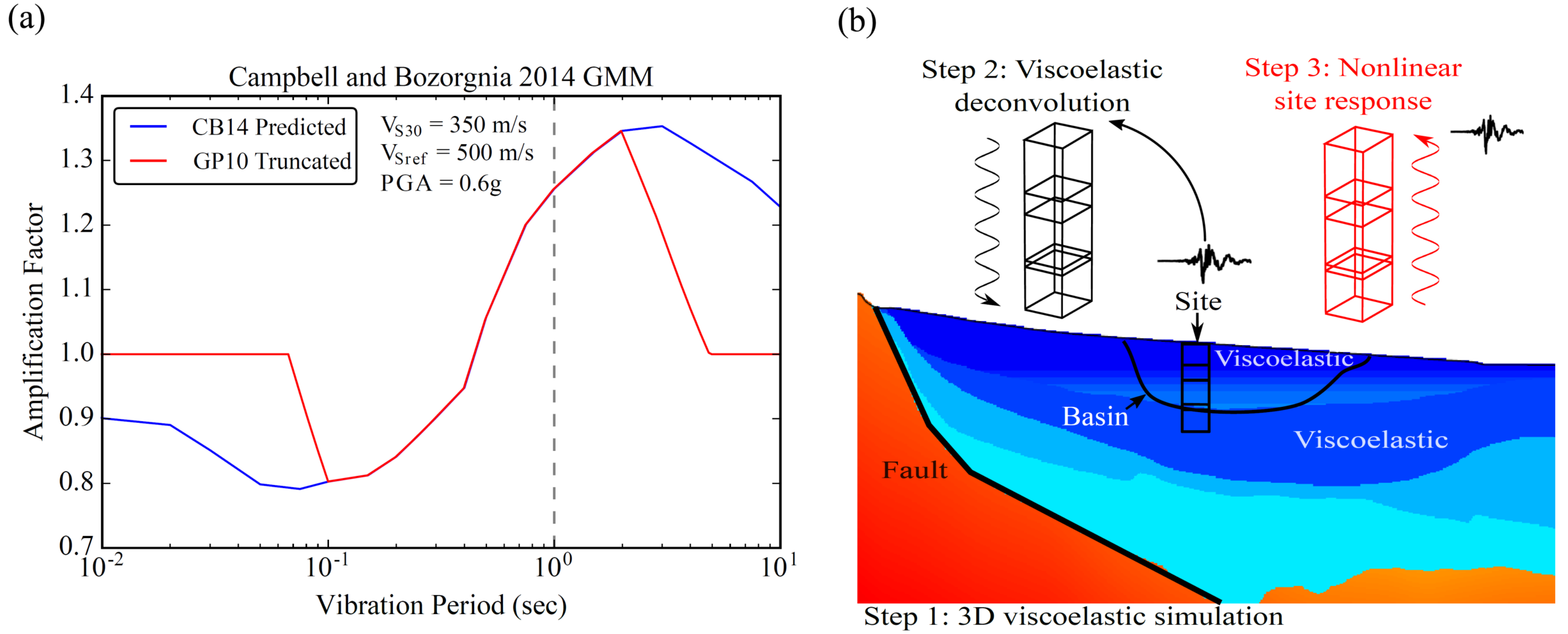

in 1: Two methods compared in this study for modelling nonlinear (a) Empirical $V_{s 30}$-based nonlinear site amplification factors rom Campbell and Bozorgnia (2014) GMM, applied to simulated ground motions in the frequency domain, and (b) Simulated ground motions propagation site response analysis.

3. Sites and Earthquakes Considered

Eleven events from the 2010-2011 Canterbury earthquake sequence with $4.8 \leq \mathrm{Mw} \leq 7.1$ were simulated by Razafindrakoto et al (2016). A detailed stations in Christchurch using these simulations as input. Figure 2 shows the upture models for all events and the locations of strong motion stations lative to the Christchurch urban area.

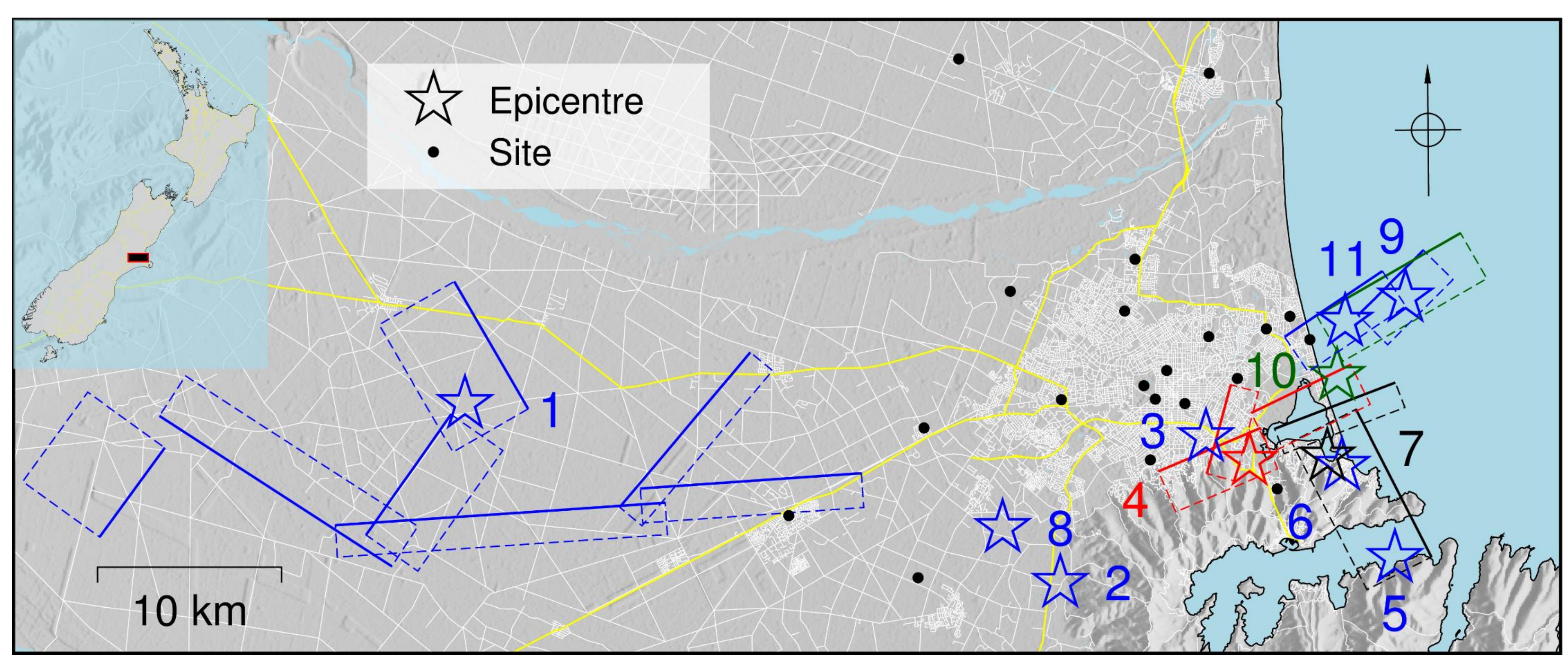

Figure 2: Earthquake rupture models for the 11 simulated earthquakes and locations of $\mathbf{2 0}$ strong motion stations analyzed.

4. Observed and Simulated Response Spectra Acceleration response spectra are compared for Ach ground motion at all sites, as illustrated for site response via the empirical $V_{530}$-based and the wave propagation methods, and viscoelastic imulations with a minimum $V s$ of $500 \mathrm{~m} / \mathrm{s}$ that neglect site effects are compared to observed ground motions. The observed-to-simulated residual of spectral accelerations is then
computed.

igure 3: Comparison between observed and

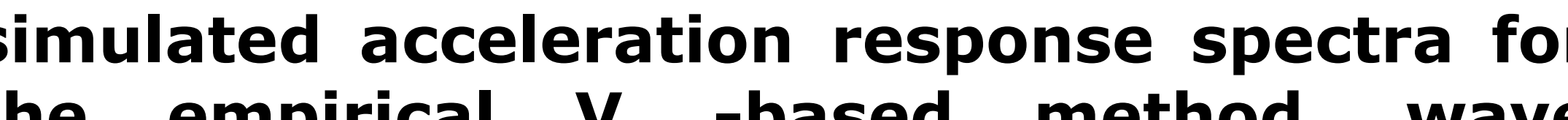
propagation site response analysis, and reference viscoelastic simulations.
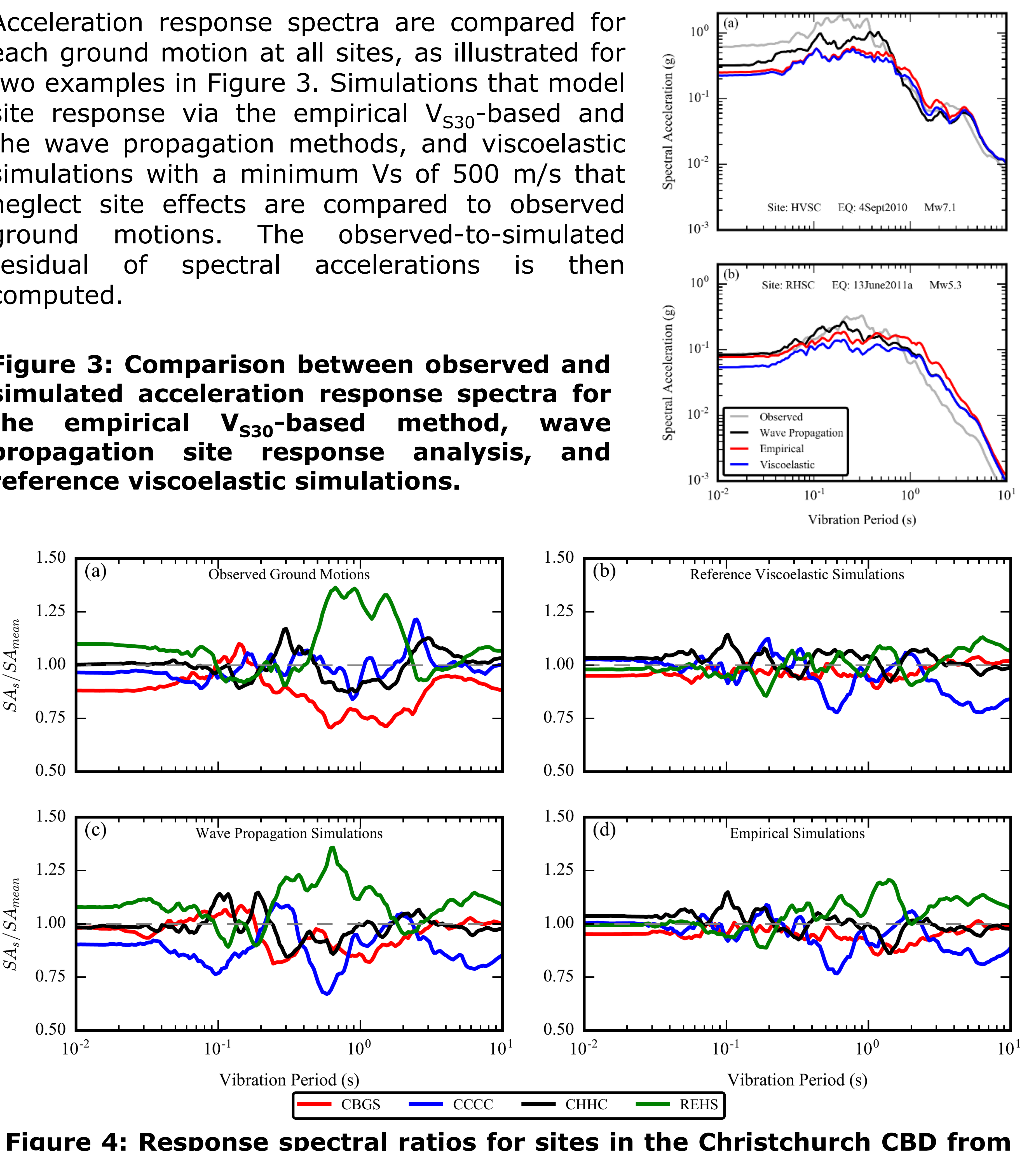

Figure 4: Response spectral ratios for sites in the Christchurch CBD from observed and simulated ground motions. The spectral ratio corresponds to the mean ratio for all 11 events where the ratio for an individual
ground motion is the spectral acceleration for a given site over the mean ground motion is the spectral

Figure 4 compares the ground surface response of nearby sites within a group response methods can capture local variability in ground motion that is attributed to near-surface site effects. Spectral ratios are computed at every site and for every event as the ratio of the surface response spectrum for each site to the geometric mean response spectrum for the full group of sites.

The wave propagation site response (Fig 4c) can capture relative features in peat site (REHS), much better than the empirical $\mathrm{V}_{\mathrm{s} 3}$-based method (Fig 4d).

\section{Systematic Prediction Residuals}

Figure 5 illustrates the systematic model bias ( $F i g 5 a$ ), and the total standard deviation, $\sigma$ (Fig $5 \mathrm{~b}$ ), in spectral acceleration prediction, as a function of analysis methods. Three average trends are identified in the results:

Reference viscoelastic simulations which ignore site effects (blue line in Fig 5) significantly underpredict spectral accelerations at periods between 0.2

The $\mathrm{V}_{\mathrm{S} 30}$-based approach significantly over-amplifies the long periods (i.e.e, $1-5 \mathrm{~s}$ ) and the wave propagation method performs better in this period

- range, suggesting that the long period component of the simulation is which the empirical amplification function is truncated (see Figure 1a) needs to be revised.

The empirical $V_{530}$-based method performs slightly better than the wave propagation approach at short periods. This is likely caused by over reference simulation which then causes further overprediction when site response is applied.

Comparison with Empirical Ground Motion Prediction

To benchmark results from simulations against the current standard of practice for ground motion prediction (i.e., empirical GMM), response spectra were and total standard deviation from the prediction via GMM. Considering both the magnitude of bias and uncertainty, it can be concluded that for periods less than 5 seconds, the physics-based and purely empirical periods predict ground motion wh the ponpasable peeformance whle for periods greater than 5 seconds the physics-based simulation methods perform significantly better.

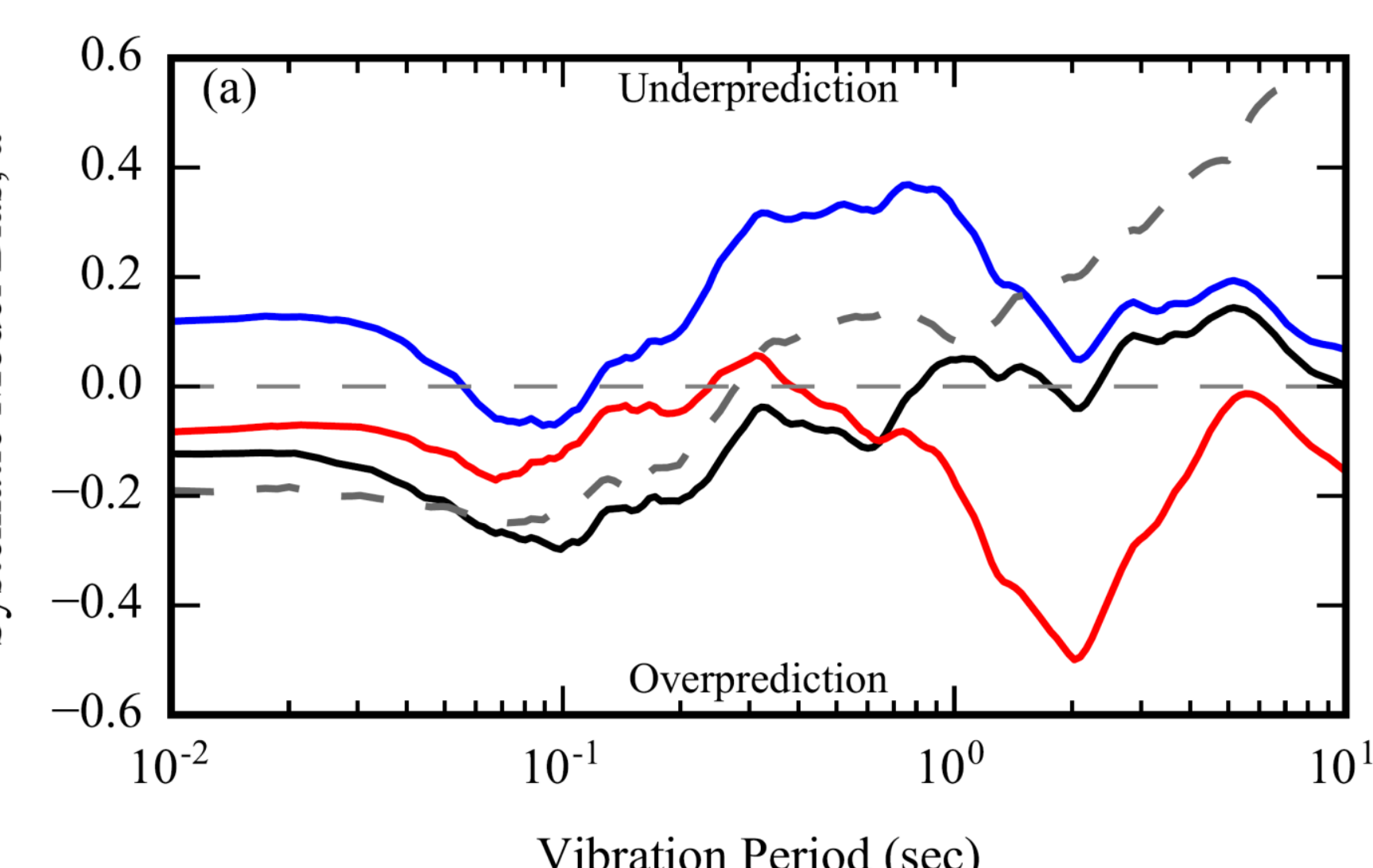

Vibration Period (seoc)

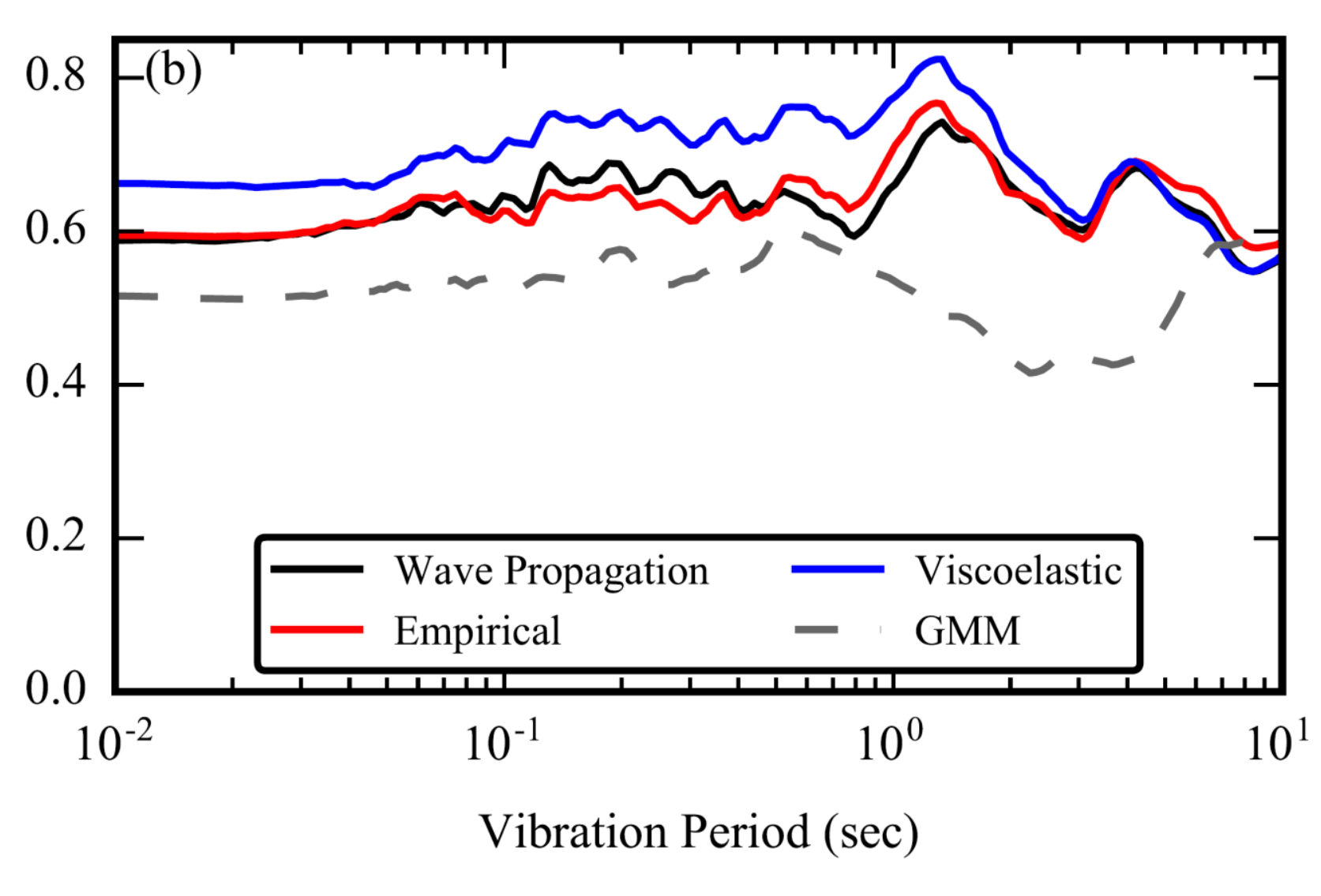

Figure 5: (a) Model bias and (b) total uncertainty from all events and wansidered for simulations that model nonlinear site effects via wave propagation site response and empirical $V_{\text {sso-based site }}$ viscoelastic condition), and purely empirical ground motion prediction 